Proyecciones

Vol. 28, No 1, pp. 75-87, May 2009.

Universidad Católica del Norte

Antofagasta - Chile

\title{
A NEW APPROACH TO ALMOST FUZZY COMPACTNESS *
}

\author{
FU-GUI SHI \\ BEIJING INSTITUTE OF TECHNOLOGY, CHINA \\ Received : February 2009. Accepted : April 2009
}

\begin{abstract}
A new definition of almost fuzzy compactness is introduced in $L$ topological spaces by means of open L-sets and their inequality when $L$ is a complete DeMorgan algebra. It can also be characterized by closed L-sets, regularly closed L-sets, regularly open L-sets and their inequalities. When $L$ is a completely distributive DeMorgan algebra, its many characterizations are presented.
\end{abstract}

Keywords : L-topology, fuzzy compactness, almost fuzzy compactness, almost continuous, weakly continuous

Subjclass [2000] : 03E72, 54A40, 54D35

\footnotetext{
${ }^{*}$ The project is supported by the National Natural Science Foundation of China (10371079) and the Basic Research Foundation of Beijing Institute of Technology.
} 


\section{Introduction}

Almost compactness has also been generalized to $L$-topological spaces by many authors (see $[2,3,4,6,9,10,14,15,16]$ ). These notions of almost fuzzy compactness rely on the structure of the basis lattice $L$, where $L=[0,1]$ or $L$ is a completely distributive DeMorgan algebra. In [21], a new definition of fuzzy compactness was presented in $L$-fuzzy topological spaces by means of open $L$-sets and their inequality.

In this paper, based on [19, 21], we shall introduce a new definition of almost fuzzy compactness in $L$-topological spaces. When $L$ is a completely distributive DeMorgan algebra, its many characterizations are presented. From these characterizations we know that it is a generalization of the notion of almost fuzzy compactness in $[3,9]$.

\section{Preliminaries}

Throughout this paper, $\left(L, \bigvee, \bigwedge,^{\prime}\right)$ is a complete DeMorgan algebra and $X$ is a nonempty set. $L^{X}$ is the set of all $L$-fuzzy sets (or $L$-sets for short) on $X$. The smallest element and the largest element in $L^{X}$ are denoted by $\underline{0}$ and $\underline{1}$.

An element $a$ in $L$ is called a prime element if $a \geq b \wedge c$ implies $a \geq b$ or $a \geq c . a$ in $L$ is called a co-prime element if $a^{\prime}$ is a prime element [7]. The set of non-unit prime elements in $L$ is denoted by $P(L)$. The set of non-zero co-prime elements in $L$ is denoted by $M(L)$.

The binary relation $\prec$ in $L$ is defined as follows: for $a, b \in L, a \prec b$ if and only if for every subset $D \subseteq L$, the relation $b \leq \sup D$ always implies the existence of $d \in D$ with $a \leq d$ [5]. In a completely distributive DeMorgan algebra $L$, each element $b$ is a supremum of $\{a \in L \mid a \prec b\}$. In the sense of [11,23], $\{a \in L \mid a \prec b\}$ is the greatest minimal family of $b$, denoted by $\beta(b)$. Moreover for $b \in L$, define $\alpha(b)=\left\{a \in L \mid a^{\prime} \prec b^{\prime}\right\}$ and $\alpha^{*}(b)=\alpha(b) \cap P(L)$.

For $a \in L$ and $A \in L^{X}$, we use the following notations in [20].

$$
\begin{aligned}
& A^{(a)}=\{x \in X \mid A(x) \not \leq a\}, \quad A_{(a)}=\{x \in X \mid a \in \beta(A(x))\}, \\
& A_{[a]}=\{x \in X \mid A(x) \geq a\} .
\end{aligned}
$$

An $L$-topological space (or $L$-space for short) is a pair $(X, \mathcal{T})$, where $\mathcal{T}$ is a subfamily of $L^{X}$ which contains $\underline{0}, \underline{1}$ and is closed for any suprema and finite infima. $\mathcal{T}$ is called an $L$-topology on $X$. Each member of $\mathcal{T}$ is called an open $L$-set and its quasi-complement is called a closed $L$-set.

Definition $2.1([\mathbf{1 1}, \mathbf{2 3}])$. For a topological space $(X, \tau)$, let $\omega_{L}(\tau)$ denote the family of all the lower semi-continuous maps from $(X, \tau)$ to $L$, i.e., 
$\omega_{L}(\tau)=\left\{A \in L^{X} \mid A^{(a)} \in \tau, a \in L\right\}$. Then $\omega_{L}(\tau)$ is an L-topology on $X$, in this case, $\left(X, \omega_{L}(\tau)\right)$ is called topologically generated by $(X, \tau)$.

Definition $2.2([\mathbf{1 1}, \mathbf{2 3}])$. An $L$-space $(X, \mathcal{T})$ is called weakly induced if $\forall a \in L, \forall A \in \mathcal{T}$, it follows that $A^{(a)} \in[\mathcal{T}]$, where $[\mathcal{T}]$ denotes the topology formed by all crisp sets in $\mathcal{T}$.

It is obvious that $\left(X, \omega_{L}(\tau)\right)$ is weakly induced.

Lemma 2.3 ([20]). Let $(X, \mathcal{T})$ be a weakly induced $L$-space, $a \in L, A \in$ $\mathcal{T}$. Then $A_{(a)}$ is an open $L$-set in $[\mathcal{T}]$. $\Phi$.

For a subfamily $\Phi \subseteq L^{X}, 2^{(\Phi)}$ denotes the set of all finite subfamilies of

Definition $2.4([19,21])$. Let $(X, \mathcal{T})$ be an $L$-space. $G \in L^{X}$ is called fuzzy compact if for every family $\mathcal{U} \subseteq \mathcal{T}$, it follows that

$$
\bigwedge_{x \in X}\left(G^{\prime}(x) \vee \bigvee_{A \in \mathcal{U}} A(x)\right) \leq \bigvee_{\mathcal{V} \in 2(\mathcal{U})} \bigwedge_{x \in X}\left(G^{\prime}(x) \vee \bigvee_{A \in \mathcal{V}} A(x)\right) .
$$

Lemma $2.5([\mathbf{1 9}, \mathbf{2 1}])$. Let $L$ be a complete Heyting algebra, $f: X \rightarrow Y$ be a map, $f_{L}: L^{X} \rightarrow L^{Y}$ is the extension of $f$, then for any family $\mathcal{P} \subseteq L^{Y}$, we have:

$$
\bigvee_{y \in Y}\left(f_{L}^{\rightarrow}(G)(y) \wedge \bigwedge_{B \in \mathcal{P}} B(y)\right)=\bigvee_{x \in X}\left(G(x) \wedge \bigwedge_{B \in \mathcal{P}} f_{L}^{\leftarrow}(B)(x)\right)
$$

Definition 2.6 $([\mathbf{1}])$. Let $\left(X, \mathcal{T}_{1}\right)$ and $\left(Y, \mathcal{T}_{2}\right)$ be two L-spaces. A map $f:\left(X, \mathcal{T}_{1}\right) \rightarrow\left(Y, \mathcal{T}_{2}\right)$ is called

(1) almost continuous if $f_{L}^{\leftarrow}(G) \in \mathcal{T}_{1}$ for all regularly open $L$-set $G$ in $\left(Y, \mathcal{T}_{2}\right)$

(2) weakly continuous if $f_{L}^{\leftarrow}(G) \leq \operatorname{int}\left(f_{L}^{\leftarrow}(\operatorname{cl}(G))\right)$ for every open $L$-set $G$ in $\left(Y, \mathcal{T}_{2}\right)$.

Lemma $2.7([\mathbf{1}])$. Let $\left(X, \mathcal{T}_{1}\right)$ and $\left(Y, \mathcal{T}_{2}\right)$ be two L-spaces. A map $f$ : $\left(X, \mathcal{T}_{1}\right) \rightarrow\left(Y, \mathcal{T}_{2}\right)$ is:

(1) almost continuous if and only if $f_{L}^{\leftarrow}(G)$ is closed in $\left(X, \mathcal{T}_{1}\right)$ for all regularly closed $L$-set $G$ in $\left(Y, \mathcal{T}_{2}\right)$.

(2) weakly continuous if and only if $f_{L}^{\leftarrow}(G) \geq \operatorname{cl}\left(f_{L}^{\leftarrow}(\operatorname{int}(G))\right)$ for every closed $L$-set $G$ in $\left(Y, \mathcal{T}_{2}\right)$. 
Lemma 2.8 ([1]). The closure of an open $L$-set is regularly closed and the interior of a closed $L$-set is regularly open.

Definition 2.9 ([8]). An $L$-space $(X, \mathcal{T})$ is said to be regular if every open $L$-set $G$ is a supremum of open $L$-sets whose closure is less that $G$.

\section{Definition and characterizations of almost fuzzy compact- ness}

Definition 3.1. Let $(X, \mathcal{T})$ be an $L$-space. $G \in L^{X}$ is called almost fuzzy compact if for every family $\mathcal{U} \subseteq \mathcal{T}$, it follows that

$$
\bigwedge_{x \in X}\left(G^{\prime}(x) \vee \bigvee_{A \in \mathcal{U}} A(x)\right) \leq \bigvee_{\mathcal{V} \in 2^{(\mathcal{U})}} \bigwedge_{x \in X}\left(G^{\prime}(x) \vee \bigvee_{A \in \mathcal{V}} \operatorname{cl}(A)(x)\right)
$$

Definition 3.2. Let $(X, \mathcal{T})$ be an $L$-space. $G \in L^{X}$ is called almost countably fuzzy compact if for every countable family $\mathcal{U} \subseteq \mathcal{T}$, it follows that

$$
\bigwedge_{x \in X}\left(G^{\prime}(x) \vee \bigvee_{A \in \mathcal{U}} A(x)\right) \leq \bigvee_{\mathcal{V} \in 2^{(\mathcal{U})}} \bigwedge_{x \in X}\left(G^{\prime}(x) \vee \bigvee_{A \in \mathcal{V}} \operatorname{cl}(A)(x)\right) .
$$

For an open $L$-set $A$, by $A \leq \operatorname{int}(\operatorname{cl}(A))$ we can obtain the following theorem.

Theorem 3.3. Fuzzy compactness $\Rightarrow$ almost fuzzy compactness $\Rightarrow$ almost countable fuzzy compactness.

From Definition 3.1 and Definition 3.2 we can obtain the following theorem by using quasi-complement.

Theorem 3.4. Let $(X, \mathcal{T})$ be an $L$-space. $G \in L^{X}$ is almost (countably) fuzzy compact if and only if for every (countable) family $\mathcal{P} \subseteq \mathcal{T}^{\prime}$, it follows that

$$
\bigvee_{x \in X}\left(G(x) \wedge \bigwedge_{A \in \mathcal{P}} A(x)\right) \geq \bigwedge_{\mathcal{F} \in 2^{(\mathcal{P})}} \bigvee_{x \in X}\left(G(x) \wedge \bigwedge_{A \in \mathcal{F}} \operatorname{int}(A)(x)\right)
$$

Definition 3.5 ([21]). Let $(X, \mathcal{T})$ be an $L$-space, $a \in L \backslash\{1\}$ and $G \in L^{X}$. $A$ family $\mathcal{A} \subseteq L^{X}$ is said to be 
(1) an $a$-shading of $G$ if for any $x \in X$, it follows that

$\left(G^{\prime}(x) \vee \bigvee_{A \in \mathcal{U}} A(x)\right) \not \leq a$.

(2) a strong a-shading of $G$ if $\bigwedge_{x \in X}\left(G^{\prime}(x) \vee \bigvee_{A \in \mathcal{U}} A(x)\right) \not \leq a$.

(3) an a-remote family of $G$ if for any $x \in X$, it follows that

$\left(G(x) \wedge \bigwedge_{B \in \mathcal{P}} B(x)\right) \nsupseteq a$.

(4) a strong a-remote family of $G$ if $\bigvee_{x \in X}\left(G(x) \wedge \bigwedge_{B \in \mathcal{P}} B(x)\right) \geq a$.

From Definition 3.1, Definition 3.2, Theorem 3.4 and Theorem 3.5 we immediately obtain the following result.

Theorem 3.6. Let $(X, \mathcal{T})$ be an $L$-space and $G \in L^{X}$. Then the following conditions are equivalent:

(1) $G$ is almost (countably) fuzzy compact.

(2) For any $a \in L \backslash\{1\}$, each (countable) open strong $a$-shading $\mathcal{U}$ of $G$ has a finite subfamily $\mathcal{V}$ such that $\mathcal{V}^{-}$is a strong a-shading of $G$, where $\mathcal{V}^{-}=\{\operatorname{cl}(A) \mid A \in \mathcal{V}\}$

(3) For any $a \in L \backslash\{0\}$, each (countable) closed strong a-remote family $\mathcal{P}$ of $G$ has a finite subfamily $\mathcal{F}$ such that $\mathcal{F}^{\circ}$ is a strong a-remote family of $G$, where $\mathcal{F}^{\circ}=\{\operatorname{int}(A) \mid A \in \mathcal{F}\}$.

Moreover by means of regularly open $L$-sets and regularly closed $L$ sets, we can give the following characterizations of almost (countable) fuzzy compactness.

Theorem 3.7. Let $(X, \mathcal{T})$ be an $L$-space and $G \in L^{X}$. Then the following conditions are equivalent:

(1) $G$ is almost (countably) fuzzy compact.

(2) For each (countable) family $\mathcal{U}$ of regularly open $L$-sets, it follows that

$$
\bigwedge_{x \in X}\left(G^{\prime}(x) \vee \bigvee_{A \in \mathcal{U}} A(x)\right) \leq \bigvee_{\mathcal{V} \in 2(\mathcal{U})} \bigwedge_{x \in X}\left(G^{\prime}(x) \vee \bigvee_{A \in \mathcal{V}} \operatorname{cl}(A)(x)\right) .
$$

(3) For each (countable) family $\mathcal{U}$ of regularly closed $L$-sets, it follows that

$$
\bigvee_{x \in X}\left(G(x) \wedge \bigwedge_{A \in \mathcal{U}} A(x)\right) \geq \bigwedge_{\mathcal{V} \in 2^{(\mathcal{U})}} \bigvee_{x \in X}\left(G(x) \wedge \bigwedge_{A \in \mathcal{V}} \operatorname{int}(A)(x)\right)
$$


Proof. $(2) \Leftrightarrow(3)$ is obvious. Because a regularly open $L$-set is open, we easily obtain $(1) \Rightarrow(2)$. Now we prove $(2) \Rightarrow(1)$. Suppose that $\mathcal{U}$ is a family of open $L$-sets. From Lemma 2.8 we know that $\operatorname{int}(\operatorname{cl}(A))$ is a regularly open $L$-set for each $A \in \mathcal{U}$. Hence by (2) we obtain

$$
\begin{aligned}
& \bigwedge_{x \in X}\left(G^{\prime}(x) \vee \bigvee_{A \in \mathcal{U}} A(x)\right) \\
= & \bigwedge_{x \in X}\left(G^{\prime}(x) \vee \bigvee_{A \in \mathcal{U}} \operatorname{int}(A)(x)\right) \\
\leq & \bigwedge_{x \in X}\left(G^{\prime}(x) \vee \bigvee_{A \in \mathcal{U}} \operatorname{int}(\operatorname{cl}(A))(x)\right) \\
\leq & \bigvee_{\left.\mathcal{V} \in 2^{(\mathcal{U}}\right)} \bigwedge_{x \in X}\left(G^{\prime}(x) \vee \bigvee_{A \in \mathcal{V}} \operatorname{cl}(\operatorname{int}(\operatorname{cl}(A)))(x)\right) \\
\leq & \bigvee_{\mathcal{V} \in 2^{(\mathcal{U})}} \bigwedge_{x \in X}\left(G^{\prime}(x) \vee \bigvee_{A \in \mathcal{V}} \operatorname{cl}(\operatorname{cl}(A))(x)\right) \\
= & \bigvee_{\mathcal{V} \in 2^{(\mathcal{U})}} \bigwedge_{x \in X}\left(G^{\prime}(x) \vee \bigvee_{A \in \mathcal{V}} \operatorname{cl}(A)(x)\right) .
\end{aligned}
$$

This shows that (1) is true.

Analogous to Theorem 3.6 we have the following result.

Theorem 3.8. Let $(X, \mathcal{T})$ be an $L$-space and $G \in L^{X}$. Then the following conditions are equivalent:

(1) $G$ is almost (countably) fuzzy compact.

(2) For any $a \in L \backslash\{1\}$, each (countable) regularly open strong $a$-shading $\mathcal{U}$ of $G$ has a finite subfamily $\mathcal{V}$ such that $\mathcal{V}^{-}$is a strong a-shading of $G$.

(3) For any $a \in L \backslash\{0\}$, each (countable) regularly closed strong $a$ remote family $\mathcal{P}$ of $G$ has a finite subfamily $\mathcal{F}$ such that $\mathcal{F}^{\circ}$ is a strong a-remote family of $G$.

Theorem 3.9. Let $(X, \mathcal{T})$ be a regular $L$-space and $G \in L^{X}$. Then $G$ is fuzzy compact if and only if it is almost fuzzy compact.

Proof. The necessity is obvious. Now we prove the sufficiency. Let $\left\{A_{i}\right\}_{i \in \Omega}$ be a family of open $L$-sets. By regularity of $(X, \mathcal{T})$, we know that for each $i \in \Omega$, there exists a family $\left\{B_{i j} \mid j \in \Delta_{i}\right\}$ of open $L$-sets such that $A_{i}=\bigvee_{j \in \Delta_{i}} B_{i j}$ and $\operatorname{cl}\left(B_{i j}\right) \leq A_{i}$. By almost fuzzy compactness of $G$, 
we know

$$
\begin{aligned}
\bigwedge_{x \in X}\left(G^{\prime}(x) \vee \bigvee_{i \in \Omega} A_{i}(x)\right) & =\bigwedge_{x \in X}\left(G^{\prime}(x) \vee \bigvee_{i \in \Omega} \bigvee_{j \in \Delta_{i}} B_{i j}(x)\right) \\
& \leq \bigvee_{\Gamma \in 2^{(\Omega)}} \bigvee_{\Theta_{i} \in 2^{\left(\Delta_{i}\right)}} \bigwedge_{x \in X}\left(G^{\prime}(x) \vee \bigvee_{i \in \Gamma} \bigvee_{j \in \Theta_{i}} \operatorname{cl}\left(B_{i j}\right)(x)\right) \\
& \leq \bigvee_{\Gamma \in 2^{(\Omega)}} \bigwedge_{x \in X}\left(G^{\prime}(x) \vee \bigvee_{i \in \Gamma} A_{i}(x)\right) .
\end{aligned}
$$

Therefore $G$ is fuzzy compact.

\section{Some properties of almost fuzzy compactness}

Theorem 4.1. Let $L$ be a complete Heyting algebra. If both $G$ and $H$ are almost (countably) fuzzy compact, then $G \vee H$ is almost (countably) fuzzy compact.

Proof. For any family $\mathcal{P}$ of closed $L$-sets, by Theorem 3.4 we have

$$
\begin{aligned}
& \bigvee_{x \in X}\left((G \vee H)(x) \wedge \bigwedge_{B \in \mathcal{P}} B(x)\right) \\
= & \left\{\bigvee_{x \in X}\left(G(x) \wedge \bigwedge_{B \in \mathcal{P}} B(x)\right)\right\} \vee\left\{\bigvee_{x \in X}\left(H(x) \wedge \bigwedge_{B \in \mathcal{P}} B(x)\right)\right\} \\
\geq & \left\{\bigwedge_{\mathcal{F} \in 2^{(\mathcal{P})}}^{\wedge} \bigvee_{x \in X}\left(G(x) \wedge \bigwedge_{B \in \mathcal{F}} \operatorname{int}(B)(x)\right)\right\} \vee \\
& \left\{\bigwedge_{\mathcal{F} \in 2^{(\mathcal{P})}}^{\wedge} \bigvee_{x \in X}\left(H(x) \wedge \bigwedge_{B \in \mathcal{F}} \operatorname{int}(B)(x)\right)\right\} \\
= & \bigwedge_{\mathcal{F} \in 2^{(\mathcal{P})}} \bigvee_{x \in X}\left((G \vee H)(x) \wedge \bigwedge_{B \in \mathcal{F}} \operatorname{int}(B)(x)\right) .
\end{aligned}
$$

This shows that $G \vee H$ is almost fuzzy compact.

Theorem 4.2. If $G$ is almost (countably) fuzzy compact, and $H$ is clopen, then $G \wedge H$ is almost (countably) fuzzy compact.

Proof. Since $G$ is almost fuzzy compact, for any family $\mathcal{P}$ of closed 
$L$-sets, by Theorem 3.4 we have

$$
\begin{aligned}
& \bigvee_{x \in X}\left((G \wedge H)(x) \wedge \bigwedge_{B \in \mathcal{P}} B(x)\right) \\
= & \bigvee_{x \in X}\left(G(x) \wedge \bigwedge_{B \in \mathcal{P} \bigcup\{\mathcal{H}\}} B(x)\right) \\
\geq & \bigwedge_{\mathcal{F} \in 2^{(\mathcal{P} \cup\{\mathcal{H}\})}} \bigvee_{x \in X}\left(G(x) \wedge \bigwedge_{B \in \mathcal{F}} \operatorname{int}(B)(x)\right) \\
= & \left\{\bigwedge_{\mathcal{F} \in 2^{(\mathcal{P})}} \bigvee_{x \in X}\left(G(x) \wedge \bigwedge_{B \in \mathcal{F}} \operatorname{int}(B)(x)\right)\right\} \\
= & \left\{\bigwedge_{\mathcal{F} \in 2^{(\mathcal{P})}} \bigwedge_{x \in X} \bigwedge_{\mathcal{F} \in 2^{(\mathcal{P})}} \bigvee_{x \in X}\left(G(x) \wedge \operatorname{int}(H)(x) \wedge \bigwedge_{B \in \mathcal{F}} \operatorname{int}(B)(x)\right)\right\} \\
= & \left.\bigwedge_{\mathcal{F} \in 2^{(\mathcal{P})}} \bigvee_{x \in X}\left((G \wedge) \operatorname{int}(H)(x) \wedge \bigwedge_{B \in \mathcal{F}} \operatorname{int}(B)(x)\right)\right\}
\end{aligned}
$$

This shows that $G \wedge H$ is almost fuzzy compact.

Theorem 4.3. Let $L$ be a complete Heyting algebra, and let $f:\left(X, \mathcal{T}_{1}\right) \rightarrow$ $\left(Y, \mathcal{T}_{2}\right)$ be almost continuous. If $G$ is almost (countably) fuzzy compact in $\left(X, \mathcal{T}_{1}\right)$, then so is $f_{L}(G)$ in $\left(Y, \mathcal{T}_{2}\right)$.

Proof. Suppose that $\mathcal{P}$ be a family of regularly closed $L$-sets, by Lemma 2.5 and almost fuzzy compactness of $G$, we have

$$
\begin{aligned}
& \bigvee_{y \in Y}\left(f_{L}^{\rightarrow}(G)(y) \wedge \bigwedge_{B \in \mathcal{P}} B(y)\right) \\
= & \bigvee_{x \in X}\left(G(x) \wedge \bigwedge_{B \in \mathcal{P}} f_{L}^{\leftarrow}(B)(x)\right) \\
\geq & \bigwedge_{\mathcal{F} \in 2^{(\mathcal{P})}} \bigvee_{x \in X}\left(G(x) \wedge \bigwedge_{B \in \mathcal{F}} \operatorname{int}\left(f_{L}^{\leftarrow}(B)\right)(x)\right) \\
\geq & \bigwedge_{\mathcal{F} \in 2^{(\mathcal{P})}} \bigvee_{x \in X}\left(G(x) \wedge \bigwedge_{B \in \mathcal{F}} f_{L}^{\leftarrow}(\operatorname{int}(B))(x)\right) \\
= & \bigwedge_{\mathcal{F} \in 2^{(\mathcal{P})}} \bigvee_{y \in Y}\left(f_{L}(G)(y) \wedge \bigwedge_{B \in \mathcal{F}} \operatorname{int}(B)(y)\right) .
\end{aligned}
$$

Therefore $f_{L}(G)$ is almost fuzzy compact. 
Theorem 4.4. Let $L$ be a complete Heyting algebra, and let $f:\left(X, \mathcal{T}_{1}\right) \rightarrow$ $\left(Y, \mathcal{T}_{2}\right)$ be weakly continuous. If $G$ is (countably) fuzzy compact in $\left(X, \mathcal{T}_{1}\right)$, then $f_{L}(G)$ is almost (countably) fuzzy compact in $\left(Y, \mathcal{T}_{2}\right)$.

Proof. Let $\mathcal{P}$ be a family of regularly closed $L$-sets, by Lemma 2.5 and fuzzy compactness of $G$, we have

$$
\begin{aligned}
& \bigvee_{y \in Y}\left(f_{L}^{\rightarrow}(G)(y) \wedge \bigwedge_{B \in \mathcal{P}} B(y)\right) \\
= & \bigvee_{x \in X}\left(G(x) \wedge \bigwedge_{B \in \mathcal{P}} f_{L}^{\leftarrow}(B)(x)\right) \\
\geq & \bigvee_{x \in X}\left(G(x) \wedge \bigwedge_{B \in \mathcal{P}} \operatorname{cl}\left(f_{L}^{\leftarrow}(\operatorname{int}(B))\right)(x)\right) \\
\geq & \bigwedge_{\mathcal{F} \in 2^{(\mathcal{P})}} \bigvee_{x \in X}\left(G(x) \wedge \bigwedge_{B \in \mathcal{F}} \operatorname{cl}\left(f_{L}^{\leftarrow}(\operatorname{int}(B))\right)(x)\right) \\
\geq & \bigwedge_{\mathcal{F} \in 2^{(\mathcal{P})}} \bigvee_{x \in X}\left(G(x) \wedge \bigwedge_{B \in \mathcal{F}} f_{L}^{\leftarrow}(\operatorname{int}(B))(x)\right) \\
= & \bigwedge_{\mathcal{F} \in 2^{(\mathcal{P})}} \bigvee_{y \in Y}^{\bigvee}\left(f_{L}(G)(y) \wedge \bigwedge_{B \in \mathcal{F}} \operatorname{int}(B)(y)\right) .
\end{aligned}
$$

Therefore $f_{L}(G)$ is almost fuzzy compact.

\section{Further characterizations of almost fuzzy compactness}

In this section, we assume that $L$ is a completely distributive DeMorgan algebra.

Definition 5.1 ([21]). Let $(X, \mathcal{T})$ be an $L$-space, $a \in L \backslash\{0\}$ and $G \in L^{X}$. $A$ family $\mathcal{U} \subseteq L^{X}$ is called a $\beta_{a}$-cover of $G$ if for any $x \in X$, it follows that $a \in \beta\left(G^{\prime}(x) \vee \bigvee_{A \in \mathcal{U}} A(x)\right) . \mathcal{U}$ is called a strong $\beta_{a}$-cover of $G$ if $a \in$ $\beta\left(\bigwedge_{x \in X}\left(G^{\prime}(x) \vee \bigvee_{A \in \mathcal{U}} A(x)\right)\right)$

Definition 5.2 ([21]). Let $(X, \mathcal{T})$ be an $L$-space, $a \in L \backslash\{0\}$ and $G \in L^{X}$. $A$ family $\mathcal{U} \subseteq L^{X}$ is called a $Q_{a}$-cover of $G$ if for any $x \in X$, it follows that $G^{\prime}(x) \vee \bigvee_{A \in \mathcal{U}} A(x) \geq a$.

Analogous to [21] we can obtain the following theorem. 
Theorem 5.3. Let $(X, \mathcal{T})$ be an $L$-space and $G \in L^{X}$. Then the following conditions are equivalent.

(1) $G$ is almost (countably) fuzzy compact.

(2) For any $a \in L \backslash\{0\}$ (or $a \in M(L)$ ), each (countable) closed strong a-remote family $\mathcal{P}$ of $G$ has a finite subfamily $\mathcal{F}$ such that $\mathcal{F}^{\circ}$ is an (a strong) a-remote family of $G$.

(3) For any $a \in L \backslash\{0\}$ (or $a \in M(L)$ ) and any (countable) closed strong $a$-remote family $\mathcal{P}$ of $G$, there exist a finite subfamily $\mathcal{F}$ of $\mathcal{P}$ and $b \in \beta(a)$ (or $b \in \beta^{*}(a)$ ) such that $\mathcal{F}^{\circ}$ is a (strong) b-remote family of $G$.

(4) For any $a \in L \backslash\{1\}$ (or $a \in P(L)$ ), each (countable) open strong $a$-shading $\mathcal{U}$ of $G$ has a finite subfamily $\mathcal{V}$ such that $\mathcal{V}^{-}$is an (a strong) $a$-shading of $G$.

(5) For any $a \in L \backslash\{1\}$ (or $a \in P(L)$ ) and any (countable) open strong $a$-shading $\mathcal{U}$ of $G$, there exist a finite subfamily $\mathcal{V}$ of $\mathcal{U}$ and $b \in \alpha(a)$ (or $b \in \alpha^{*}(a)$ ) such that $\mathcal{V}^{-}$is a (strong) b-shading of $G$.

(6) For any $a \in L \backslash\{0\}$ (or $a \in M(L)$ ), each (countable) open strong $\beta_{a}$-cover $\mathcal{U}$ of $G$ has a finite subfamily $\mathcal{V}$ such that $\mathcal{V}^{-}$is a (strong) $\beta_{a^{-} \text {-cover }}$ of $G$.

(7) For any $a \in L \backslash\{0\}$ (or $a \in M(L)$ ) and any (countable) open strong $\beta_{a}$-cover $\mathcal{U}$ of $G$, there exist a finite subfamily $\mathcal{V}$ of $\mathcal{U}$ and $b \in L$ (or $b \in$ $M(L))$ with $a \in \beta(b)$ such that $\mathcal{V}^{-}$is a (strong) $\beta_{b}$-cover of $G$.

(8) For any $a \in L \backslash\{0\}$ (or $a \in M(L)$ ) and any $b \in \beta(a) \backslash\{0\}$, each (countable) open $Q_{a}$-cover of $G$ has a finite subfamily $\mathcal{V}$ such that $\mathcal{V}^{-}$is a $Q_{b}$-cover of $G$.

(9) For any $a \in L \backslash\{0\} \quad$ (or $a \in M(L)$ ) and any $b \in \beta(a) \backslash\{0\} \quad$ (or $b \in$ $\beta^{*}(a)$ ), each (countable) open $Q_{a}$-cover of $G$ has a finite subfamily $\mathcal{V}$ such

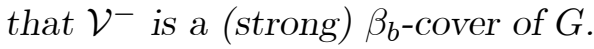

Remark 5.4. In Theorem 5.3, 'open' can be replaced by 'regularly open', and 'closed' can be replaced by 'regularly closed'.

Remark 5.5. From (2) of Theorem 5.3 we know that our notion of almost fuzzy compactness is a generalization of almost F-compactness in [3, 9].

The following theorem shows that almost (countable) fuzzy compactness is a good extension.

Theorem 5.6. Let $(X, \tau)$ be a topological space and $(X, \omega(\tau))$ be generated topologically by $(X, \tau)$. Then $(X, \omega(\tau))$ is almost (countably) fuzzy compact if and only if $(X, \tau)$ is almost (countably) compact. 
Proof. (Necessity) Let $\mathcal{A}$ be an open cover of $(X, \tau)$. Then $\left\{\chi_{A} \mid\right.$ $A \in \mathcal{A}\}$ is a family of open $L$-sets in $(X, \omega(\tau))$ with $\bigwedge_{x \in X}\left(\bigvee_{A \in \mathcal{U}} \chi_{A}(x)\right)=1$ From almost fuzzy compactness of $(X, \omega(\tau))$ we know that

$$
\bigvee_{\mathcal{V} \in 2^{(\mathcal{U})}} \bigwedge_{x \in X}\left(\bigvee_{A \in \mathcal{V}} \chi_{\mathrm{cl}(A)}(x)\right)=\bigvee_{\mathcal{V} \in 2^{(\mathcal{U})}} \bigwedge_{x \in X}\left(\bigvee_{A \in \mathcal{V}} \operatorname{cl}\left(\chi_{A}\right)(x)\right)=1
$$

This implies that there exists $\mathcal{V} \in 2^{(\mathcal{U})}$ such that $\bigwedge_{x \in X}\left(\bigvee_{A \in \mathcal{V}} \chi_{\operatorname{cl}(A)}(x)\right)=1$. Hence $\{\operatorname{cl}(A) \mid A \in \mathcal{V}\}$ is a cover of $(X, \tau)$. Therefore $(X, \tau)$ is almost compact.

(Sufficiency) Let $\mathcal{U}$ be a family of open $L$-sets in $(X, \omega(\tau))$ and let $\bigwedge_{x \in X}\left(\bigvee_{B \in \mathcal{U}} B(x)\right)=a$. If $a=0$, then obviously we have

$$
\bigwedge_{x \in X}\left(\bigvee_{B \in \mathcal{U}} B(x)\right) \leq \bigvee_{\mathcal{V} \in 2^{(\mathcal{U})}} \bigwedge_{x \in X}\left(\bigvee_{A \in \mathcal{V}} \operatorname{cl}(B)(x)\right)
$$

Now we suppose that $a \neq 0$. In this case, for any $b \in \beta(a) \backslash\{0\}$ we have

$$
b \in \beta\left(\bigwedge_{x \in X}\left(\bigvee_{B \in \mathcal{U}} B(x)\right)\right) \subseteq \bigcap_{x \in X} \beta\left(\bigvee_{B \in \mathcal{U}} B(x)\right)=\bigcap_{x \in X} \bigcup_{B \in \mathcal{U}} \beta(B(x)) .
$$

From Lemma 2.3 this implies that $\left\{B_{(b)} \mid B \in \mathcal{U}\right\}$ is an open cover of $(X, \tau)$. From almost fuzzy compactness of $(X, \tau)$ we know that there exists $\mathcal{V} \in 2^{(\mathcal{U})}$ such that $\left\{\operatorname{cl}\left(B_{(b)}\right) \mid B \in \mathcal{V}\right\}$ is a cover of $(X, \tau)$. From [17] we can obtain that $\operatorname{cl}\left(B_{(b)}\right) \subseteq \operatorname{cl}(B)_{[b]}$. This shows that $\left\{\operatorname{cl}(B)_{[b]} \mid B \in \mathcal{V}\right\}$ is a cover of $(X, \tau)$. Hence $b \leq \bigwedge_{x \in X}\left(\bigvee_{B \in \mathcal{V}} \operatorname{cl}(B)(x)\right)$. Further we have

$$
b \leq \bigwedge_{x \in X}\left(\bigvee_{B \in \mathcal{V}} \operatorname{cl}(B)(x)\right) \leq \bigvee_{\mathcal{V} \in 2^{(\mathcal{U})}} \bigwedge_{x \in X}\left(\bigvee_{B \in \mathcal{V}} \operatorname{cl}(B)(x)\right) .
$$

This implies

$$
\bigwedge_{x \in X}\left(\bigvee_{B \in \mathcal{U}} B(x)\right)=a=\bigvee\{b \mid b \in \beta(a)\} \leq \bigvee_{\mathcal{V} \in 2^{(\mathcal{U})}} \bigwedge_{x \in X}\left(\bigvee_{B \in \mathcal{V}} \operatorname{cl}(B)(x)\right) .
$$

Therefore $(X, \omega(\tau))$ is almost fuzzy compact. 


\section{References}

[1] K.K. Azad, On fuzzy semicontinuity, fuzzy almost continuity and fuzzy weakly continuity, J. Math. Anal. Appl. 82, pp. 14-32, (1981).

[2] A. Bülbül and M.W. Warner, Some good dilutions of fuzzy compactness, Fuzzy Sets and Systems 51, pp. 111-115, (1992).

[3] S.L. Chen, Almost F-compactness in $L$-fuzzy topological spaces, $J$. Northeastern Math. 7, pp. 4428-4432, (1991).

[4] A.D. Concilio and G. Gerla, Almost compactness in fuzzy topological spaces, Fuzzy Sets and Systems 13, pp. 187-192, (1984).

[5] P. Dwinger, Characterizations of the complete homomorphic images of a completely distributive complete lattice I, Indagationes Mathematicae (Proceedings) 85, pp. 403-414, (1982).

[6] A.Haydar Es, Almost compactness and near compactness in fuzzy topological spaces, Fuzzy Sets and Systems 22, pp. 289-295, (1987).

[7] G. Gierz, et al., A compendium of continuous lattices, Springer Verlag, Berlin, (1980).

[8] B. Hutton, I. Reilly, Separation axioms in fuzzy topological spaces, Fuzzy Sets and Systems 3, pp. 93-104, (1980).

[9] C. Kaur, A. Sharfuddin, On almost compactness in the fuzzy setting, Fuzzy Sets and Systems 125, pp. 163-165, (2002).

[10] S.R.T. Kudri and M.W. Warner, Some good $L$-fuzzy compactnessrelated concepts and their properties I, Fuzzy Sets and Systems 76, pp. 141-155, (1995).

[11] Y.M. Liu and M.K. Luo, Fuzzy topology, Singapore: World Scientific, (1997).

[12] R. Lowen, Fuzzy topological spaces and fuzzy compactness. J. Math. Anal. Appl. 56, pp. 621-633, (1976).

[13] R. Lowen, A comparision of different compactness notions in fuzzy topological spaces, J. Math. Anal. Appl. 64, pp. 446-454, (1978). 
[14] H. Meng and G.W. Meng, Almost N-compact sets in $L$-fuzzy topological spaces, Fuzzy Sets and Systems, 91, pp. 115-122,(1997).

[15] M.N. Mukherjee and S.P. Sinha, Almost compact fuzzy sets in fuzzy topological spaces, Fuzzy Sets and Systems, 38, pp. 389-396, (1990).

[16] M.N. Mukherjee and R.P. Chakraborty, On fuzzy almost compact spaces, Fuzzy Sets and Systems, 98, pp. 207-210, (1998).

[17] F.-G. Shi, Theory of $L_{\beta}$-nested sets and $L_{\alpha}$-nested and their applications, Fuzzy Systems and Mathematics, 4, pp. 65-72, (1995)(in Chinese).

[18] F.-G. Shi, C.Y. Zheng, O-convergence of fuzzy nets and its applications, Fuzzy Sets and Systems 140, pp. 499-507, (2003).

[19] F.-G. Shi, Countable compactness and the Lindelöf property of $L$-fuzzy sets, Iranian Journal of Fuzzy Systems 1, pp. 79-88, (2004).

[20] F.-G. Shi, A new notion of fuzzy compactness in $L$-topological spaces, Information Sciences 173, pp. 35-48, (2005).

[21] F.-G. Shi, A new definition of fuzzy compactness, Fuzzy Sets and Systems 158, pp., 1486-1495, (2007).

[22] G.J. Wang, A new fuzzy compactness defined by fuzzy nets, J. Math. Anal. Appl. 94, pp., 1-23, (1983).

[23] G.J. Wang, Theory of $L$-fuzzy topological space, Shaanxi Normal University Press, Xi'an, (1988) (in Chinese).

[24] J.J. Xu, On fuzzy compactness in $L$-fuzzy topological spaces, Chinese Quarterly Journal of Mathematics 2, pp., 104-105, (1990)(in Chinese).

[25] D.S. Zhao, The N-compactness in $L$-fuzzy topological spaces, J. Math. Anal. Appl. 128, pp., 64-70, (1987).

\section{Fu-Gui Shi}

Department of Mathematics

Beijing Institute of Technology

Beijing 100081,

P. R. China

e-mail : fuguishi@bit.edu.cn or f.g.shi@263.net 